\title{
IKKe knockout alleviates angiotensin II-induced apoptosis and excessive autophagy in vascular smooth muscle cells by regulating the ERK1/2 pathway
}

\author{
GANYI CHEN* ${ }^{*}$ YUEYUE XU* ${ }^{*}$ YIWEI YAO, YIDE CAO, YAFENG LIU, \\ HAO CHAI, WEN CHEN and XIN CHEN \\ Department of Thoracic and Cardiovascular Surgery, Nanjing First Hospital, \\ Nanjing Medical University, Nanjing, Jiangsu 210006, P.R. China
}

Received October 18, 2020; Accepted June 18, 2021

DOI: $10.3892 /$ etm.2021.10485

\begin{abstract}
Inhibitor of nuclear factor- $\kappa \mathrm{B}$ kinase subunit $\varepsilon$ $(\mathrm{IKK} \varepsilon)$ is an important signal regulator in the formation of abdominal aortic aneurysm (AAA). However, the underlying mechanism remains to be elucidated. Therefore, the present study aimed to investigate the mechanism underlying IKKe function in AAA formation by studying apoptosis and autophagy in angiotensin II (Ang II)-induced vascular smooth muscle cells (VSMCs). AngII was used to stimulate VSMCs for $24 \mathrm{~h}$ to simulate the process of AAA formation. VSMCs were transfected with IKKe small interfering RNA to investigate the effect of IKKe on AAA formation, cell apoptosis and autophagy. IKK $\varepsilon$ deficiency led to reduced mitochondrial damage and apoptosis in VSMCs in the early stage of apoptosis in vitro, as demonstrated using a JC-1 probe. IKKe deficiency also reduced autophagy and decreased the formation of autophagic vacuoles in VSMCs, demonstrated using transmission electron microscopy. The decrease in apoptosis caused by IKKe knockdown was reversed when the autophagic flow was blocked using bafilomycin A1. Western blot analysis further revealed that IKKE deficiency negatively regulated the ERK1/2 signaling pathway to reduce autophagy. Collectively, the results of the present study revealed that IKKe played a key role in apoptosis by inducing excessive autophagy, thereby potentially contributing to AAA formation. These findings further revealed the mechanism underlying IKKe function in the formation of AAA.
\end{abstract}

Correspondence to: Dr Wen Chen or Professor Xin Chen, Department of Thoracic and Cardiovascular Surgery, Nanjing First Hospital, Nanjing Medical University, 68 Changle Road, Nanjing, Jiangsu 210006, P.R. China

E-mail: cxacf@163.com

E-mail: stevecx@njmu.edu.cn

${ }^{*}$ Contributed equally

Key words: abdominal aortic aneurysm, inhibitor of nuclear factor- $\kappa \mathrm{B}$ kinase subunit $\varepsilon$, autophagy, apoptosis, vascular smooth muscle cells

\section{Introduction}

Abdominal aortic aneurysm (AAA) is a complicated and dangerous cardiovascular disease characterized by high morbidity and mortality globally $(1,2)$. In developing countries, the incidence of AAA rises to 3-4\% in people aged over 65, and rupture of AAA causes $~ 4,500$ deaths in the United States $(1,2)$. Most AAA-related deaths are attributed to AAA rupture (3). Currently, surgery is the only treatment option for AAA; however, the benefits of surgery are limited by the aortic diameter (4). Moreover, AAA pathogenesis during formation and development is yet to be fully elucidated. Apoptosis of vascular smooth muscle cells (VSMCs) aggravates AAA progression at the histopathological level (5). Thus, it is necessary to explore new therapeutic approaches, such as inhibition of apoptosis in VSMCs, in order to minimize AAA progression.

Findings of previous studies report that AAA and atherosclerosis share similar histopathological characteristics $(6,7)$. Inhibitor of nuclear factor- $\kappa \mathrm{B}$ kinase subunit $\varepsilon$ (IKK $\varepsilon$ ) regulates a variety of pathophysiological processes associated with the occurrence of atherosclerosis, such as morphological changes and lipid accumulation in the aorta (8). It is also an important signal regulator that plays a crucial role in tumorigenesis, inflammation, metabolic disorders and the reduction-oxidation process (9). AAA is characterized by inflammatory cell infiltration and apoptosis of VSMCs (5). Therefore, it was inferred that IKKe plays an important role in AAA development. A previous study revealed that IKKe expression is significantly increased in patients with AAA (10). The results of a another study indicated that the lack of IKKe reduced AAA formation by reducing apoptosis and inflammation in the abdominal aorta of mice (11). However, the mechanism underlying IKKe function remains unclear.

Autophagy and apoptosis are two key forms of cell death (12). In the cardiovascular system, autophagy protects blood vessels from dysfunction (13). However, excessive autophagy leads to cell death during the pathological state under ER stress (14). Myocardin regulates the apoptosis of VSMCs by regulating autophagy to promote the occurrence of aortic aneurysms (15). A previous study has reported that 
the targeted knockdown of autophagy-related protein (ATG)7 or the autophagy inhibitor 3-methyladenine inhibited caspase activation and reduced apoptosis (16). Furthermore, a recent study postulated that $\mathrm{IKK} \varepsilon$ is an autophagy-activating gene in breast cancer (17). Multiple autophagy-related genes, such as ATG5 and ATG7 are markedly upregulated in human aortic aneurysm disease (18). The present study investigated the effects of IKKe on autophagy and apoptosis in a VSMC model of AAA.

In the present study, loss of IKKe ameliorated angiotensin II (AngII)-induced cellular AAA model by inhibiting autophagy and apoptosis in VSMCs. This phenomenon was partially dependent on activated ERK1/2 signaling.

\section{Materials and methods}

Cell line and culture. Mouse thoracic aortic vascular smooth muscle cell (VSMCs; cat. no. CRL-2797) were obtained from the American Type Culture Collection and cultured in DMEM (Gibco; Thermo Fisher Scientific, Inc.) with 10\% FBS (Gibco; Thermo Fisher Scientific, Inc.) and 1\% antibiotic-antimycotic solution (100 U/ml penicillin and $100 \mu \mathrm{g} / \mathrm{ml}$ streptomycin). Cells were incubated in a humidified incubator at $37^{\circ} \mathrm{C}$ with $5 \% \mathrm{CO}_{2}$ (Thermo Fisher Scientific, Inc.). VSMCs were passaged 3-5 times and seeded in six-well plates at a density of $1.0 \times 10^{4}$ cells/well. The following day, cells were treated with AngII ( $1 \mu \mathrm{mol} / \mathrm{l}$; Sigma Aldrich; Merck KGaA) for $24 \mathrm{~h}$.

Transfection. VSMCs at $60 \%$ confluence were cultured in six-well plates and transfected with IKKe small interfering (si) RNA (20 $\mu \mathrm{mol} / 1$; Shanghai GenePharma Co., Ltd.) for $6 \mathrm{~h}$ using Lipofectamine ${ }^{\circledR} 3000$ (Invitrogen; Thermo Fisher Scientific, Inc.) according to the manufacturer's protocol. The siRNA was used to infect cells for $6 \mathrm{~h}$ at $37^{\circ} \mathrm{C}$ and cell medium was replaced with complete culture medium. The following day, cells were exposed to AngII for $24 \mathrm{~h}$. The sequences were as follows: A sense, 5'-GCAUACUGAUGACCUGCUATT-3' and antisense, 5'-UAGCAGGUCAUCAGUAUGCTT-3'; b sense, 5'-CCC ACAACACGAUUGCCAUTT-3' and antisense 5'-AUGGCA AUCGUGUUGUGGGTT-3'; c sense, 5'-GCAACCUAUGGC UCCUCAUTT-3' and antisense, 5'-AUGAGGAGCCAUAGG UUGCTT-3' and the control sense, 5'-UUCUCCGAACGU GUCACGUTT-3' and antisense, 5'-ACGUGACACGUUCGG AGAATT-3'.

Western blot analysis. Total proteins of VSMCs were extracted using RIPA buffer (Beyotime Institute of Biotechnology) and their concentrations were determined using the BCA protein assay kit (cat. no. KGP902; Nanjing KeyGen Biotech Co., Ltd.). The proteins (30 $\mu \mathrm{g}$ per lane) were then separated via 10 and $12 \%$ SDS-PAGE, and the resultant bands were transferred onto PVDF membranes (Sigma-Aldrich; Merck KGaA). The membranes were blocked with 5\% skimmed milk powder for $1 \mathrm{~h}$ at room temperature and subsequently incubated with primary antibodies at $4^{\circ} \mathrm{C}$ overnight. The antibodies included anti-IKKe (1:1,000; cat. no. 3416S; Cell Signaling Technology, Inc.), anti-LC3B (1:2,000; cat. no. ab51520; Abcam), anti-P62 (1:1,000; cat. no. 23214S; Cell Signaling Technology, Inc.), anti-ATG7 (1:1,000; cat. no. 8558S; Cell Signaling Technology, Inc.), anti-Bax (1:1,000; cat. no. 2772S;
Cell Signaling Technology, Inc.), anti-cleaved (c)-caspase-3 (1:1,000; cat. no. 9661S; Cell Signaling Technology, Inc.), anti-caspase-9 (1:1,000; cat. no. 9508T; Cell Signaling Technology, Inc.), anti-p38 (1:1,000; cat. no. 9212S; Cell Signaling Technology, Inc.), anti-phosphorylated (p)-p38 (1:1,000; cat. no. 4511S; Cell Signaling Technology, Inc.), anti-MEK1/2 (1:1,000; cat. no. 9122S; Cell Signaling Technology, Inc.), anti-p-MEK1/2 (1:1,000; cat. no. 9154S; Cell Signaling Technology, Inc.), anti-ERK1/2 (1:1,000; cat. no.4695S; Cell Signaling Technology, Inc.), anti-p-ERK1/2 (1:1,000; cat. no. 4370S; Cell Signaling Technology, Inc.) and anti-GAPDH antibody (1:5,000; cat. no. 8884S; Cell Signaling Technology, Inc.). The PVDF membranes were then incubated with secondary antibodies, including HRP-anti-mouse IgG (1:5,000; cat. no. HRP-60004; ProteinTech Group, Inc.) and HRP-anti-Rabbit IgG (1:5,000; cat. no. 7074P2; Cell Signaling Technology, Inc.) for $1 \mathrm{~h}$ at room temperature. The protein bands were subsequently visualized using chemiluminescent HRP Substrate (cat. no. P90720; MilliporeSigma) and captured on a Hyperfilm (Amersham; Cytiva), and the results were analyzed using ImageJ software (version 1.8.0; National Institutes of Health) for semi-quantitation of the mean gray value of each blot.

Flow cytometry analysis. Apoptosis was measured using the Annexin-V/PI double staining method. VSMCs were seeded in six-well plates at a density of $1.0 \times 10^{4}$ cells/well and treated with AngII for $24 \mathrm{~h}$, washed with PBS, and subsequently trypsinized to obtain a single-cell suspension. The Annexin V-FITC Apoptosis Detection Kit (Nanjing KeyGen Biotech Co., Ltd.) was used to stain the cells according to the manufacturer's protocol. Apoptotic cells were subsequently evaluated using a flow cytometer (BD FACSCalibur ${ }^{\mathrm{TM}}$; BD Biosciences). The results were analyzed using ModFit LT version 5.0 (Verity Software House, Inc.).

Transmission electron microscopy. VSMCs were centrifuged to form clusters at $67 \mathrm{x} \mathrm{g}$ and room temperature for $5 \mathrm{~min}$, and subsequently fixed with $2.5 \%$ glutaraldehyde at $4^{\circ} \mathrm{C}$ overnight. Cells were further fixed in $1 \%$ buffered osmium tetroxide and dehydrated in graded ethanol. Cluster sections $(60-70 \mathrm{~nm})$ were double stained with $2 \%$ uranyl acetate for $2 \mathrm{~h}$ at room temperature and lead citrate for $5 \mathrm{~min}$ at room temperature. The samples were examined under a JEOL JEM-1010 transmission electron microscope (JEOL, Ltd.). The autophagosomes were manually counted.

Mitochondrial membrane potential analysis. The JC-1 probe (Beyotime Institute of Biotechnology) was employed to measure mitochondrial depolarization in VSMCs. The cells were first cultured with AngII in six-well plates at a density of $1.0 \times 10^{4}$ cells/well for $24 \mathrm{~h}$ and subsequently incubated with an equal volume of JC-1 staining solution $(5 \mathrm{pg} / \mathrm{ml})$ at $37^{\circ} \mathrm{C}$ for $20 \mathrm{~min}$. Cells were then rinsed twice with PBS, followed by monitoring of the mitochondrial membrane potentials by determining the relative amounts of dual emissions from mitochondrial JC-1 monomers and aggregates. Mitochondrial membrane potentials were monitored using an Olympus fluorescent microscope (Olympus Corporation) under Argon-ion $488 \mathrm{~nm}$ laser excitation. An increase in the ratio 
of green/red (488/594 nm) fluorescence intensity indicated mitochondrial depolarization. The results were analyzed using ImageJ software (version 1.8.0; National Institutes of Health).

Autophagic flux analysis. VSMCs were first treated with bafilomycin A1 (Baf-A1; $400 \mathrm{nM}$; Selleck Chemicals) for $4 \mathrm{~h}$ prior to AngII treatment as previously described, to block autophagosome-lysosome fusion. Autophagic flux was measured via western blot analysis, as previously described.

Statistical analysis. Data are presented as the mean \pm standard error. Differences between groups were determined using ANOVA followed by Tukey's post hoc test. Comparisons between two groups were performed using a paired Student's t-test. All statistical analyses were performed using GraphPad Prism 6.0 software (GraphPad Software, Inc.). $\mathrm{P}<0.05$ was considered to indicate a statistically significant difference.

\section{Results}

IKKe knockdown attenuates AngII-induced apoptosis in $V S M C s$. IKKe deficiency has been indicated to attenuate AAA formation in mice by inhibiting apoptosis (11). In the present study, VSMCs were transfected with siRNA to knock down $\mathrm{IKK} \varepsilon$, and were subsequently exposed to AngII for $24 \mathrm{~h}$ to elucidate the role of $\mathrm{IKK} \varepsilon$ in apoptosis in vitro. The sequences of IKKe siRNA with the highest knockdown efficiency were selected (Fig. S1A and B). IKKe knockdown efficiency in VSMCs was 40-60\% compared with the control group. (Fig. 1A and B). Western blot analysis revealed that IKKe knockdown reduced the expression of caspase-9, c-caspase-3 and Bax in VSMCs after induction with AngII (Fig. 1A and B). The analysis of the apoptosis of VSMCs detected by flow cytometry revealed that IKKe knockdown reduced the apoptosis of VSMCs following AngII treatment (Fig. 1C and D). Furthermore, JC-1 staining revealed that IKKe deficiency reduced mitochondrial damage after AngII treatment, which occurs at an early stage of apoptosis (19) (Fig. 1E and F). Collectively, the results of the present study demonstrated that IKKe knockdown reduced apoptosis of VSMCs at an early stage with AngII treatment.

IKKE knockdown attenuates AngII-induced excessive autophagy in VSMCs. Autophagy in VSMCs was further investigated to verify the role of IKKe on apoptosis in VSMCs. Western blot analysis of LC3B, ATG7 and P62 revealed that IKKe knockdown reduced autophagy in AngII-induced VSMCs (Fig. 2A and B). Transmission electron microscopy further revealed that autophagic vacuoles were significantly reduced in AngII-induced VSMCs in the SiIKKe group compared with the control group (Fig. 2C and D).

IKKE plays an important role in the association of autophagy and apoptosis. To detect the autophagy flow in vitro, Baf-A1 was used to block the autophagy flow in AngII-induced VSMCs. The expression levels of LC3B and P62 in the VSMCs were significantly increased following autophagy blocking with Baf-A1 and AngII compared with the AngII group, indicating that the autophagic process was active in the
AngII-induced VSMC autophagosomes (20) (Fig. 3A and B). A smaller decrease in the expression of LC3B and P62 was demonstrated in the IKKe deficiency with Baf-A1 + AngII group compared with the control + AngII + Baf-A1 group, indicating that $\mathrm{IKK} \varepsilon$ potentially played a role in autophagy (Fig. 3A and B). Notably, there was no significant difference in the expression levels of caspase- 9 and Bax in the SiIKKe + AngII + Baf-A1 group compared with the control + AngII + Baf-A1 group (Fig. 3C and D). Thus, it was hypothesized that $\mathrm{IKK} \varepsilon$ increased apoptosis by promoting autophagy in VSMCs.

IKKE regulates autophagy and apoptosis via the ERK1/2 signaling pathway. The activation of the ERK1/2 pathway in VSMCs was assessed to further investigate the mechanism underlying IKKe in autophagy regulation in vitro. The level of p-ERK1/2 was decreased in SiIKKe cells exposed to AngII compared with control cells exposed to AngII (Fig. 4A and B). The phosphorylation levels of MEK1/2 and p38 were investigated, and no significant difference was demonstrated in the phosphorylation of MEK1/2 and p38 in VSMCs with AngII treatment (Fig. S2A and B). These results indicated that IKKe knockdown reduced autophagy and apoptosis by inhibiting the ERK1/2 signaling pathway.

\section{Discussion}

AAA is a disease associated with serious complications such as the rupture of aneurysm and death, and is more severe than common heart disease (21). However, the specific pathogenesis of AAA is yet to be elucidated. The results of the present study demonstrated the important role of IKKe in autophagy, apoptosis and AAA development. Knockdown of IKKe attenuated AngII-induced apoptosis and excessive autophagy in VSMCs. IKKe also increased apoptosis by promoting the autophagy of VSMCs. Moreover, IKKe knockdown reduced autophagy and apoptosis by inhibiting the ERK1/2 signaling pathway.

Apoptosis of VSMCs is a key biomarker of AAA formation (5). The results of a previous study demonstrated that IKKe played an important role in regulating apoptosis of VSMCs in mice (11). In the present study, the in vitro results were consistent with previous findings (8). It has been previously revealed that when the membrane potential of mitochondria decreases, the permeability of the mitochondrial membrane increases and the proapoptotic factors are released into the cytoplasm (22). In the present study, IKKe deficiency reduced mitochondrial damage and apoptosis in vitro, indicating that IKK $\varepsilon$ regulated the apoptosis of VSMCs via the mitochondrial apoptosis pathway.

Activated autophagy is generally considered a cell protection mechanism due to the promotion of cell survival (23). However, excessive activation of autophagy has been indicated to result in autophagic cell death (24). Functional autophagy is essential for cardiac homeostasis (25); however uncontrolled autophagy induction in ischemia-reperfusion injury response resulted in excessive cardiomyocyte apoptosis, thereby aggravating the injury (26). VSMCs may exhibit excessive autophagy leading to cell death when exposed to severe stimuli (27). This mechanism serves a role in the occurrence of a number of vascular diseases, such as atherosclerosis. A previous study postulated that suppressing autophagy inhibited AAA development (28). 


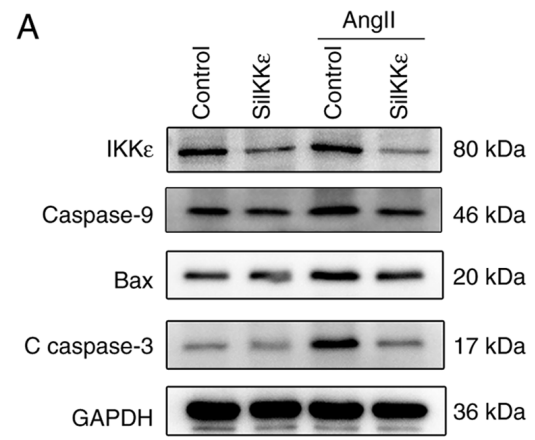

$\square$ Control+Angll
$\square$ SilKK\&+Angll

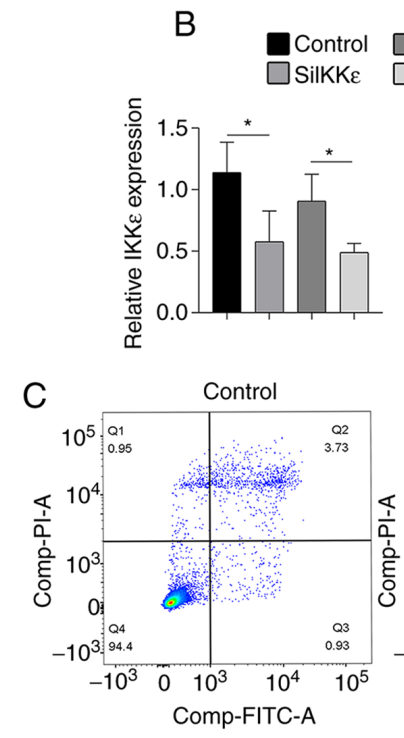

$\mathrm{D}$
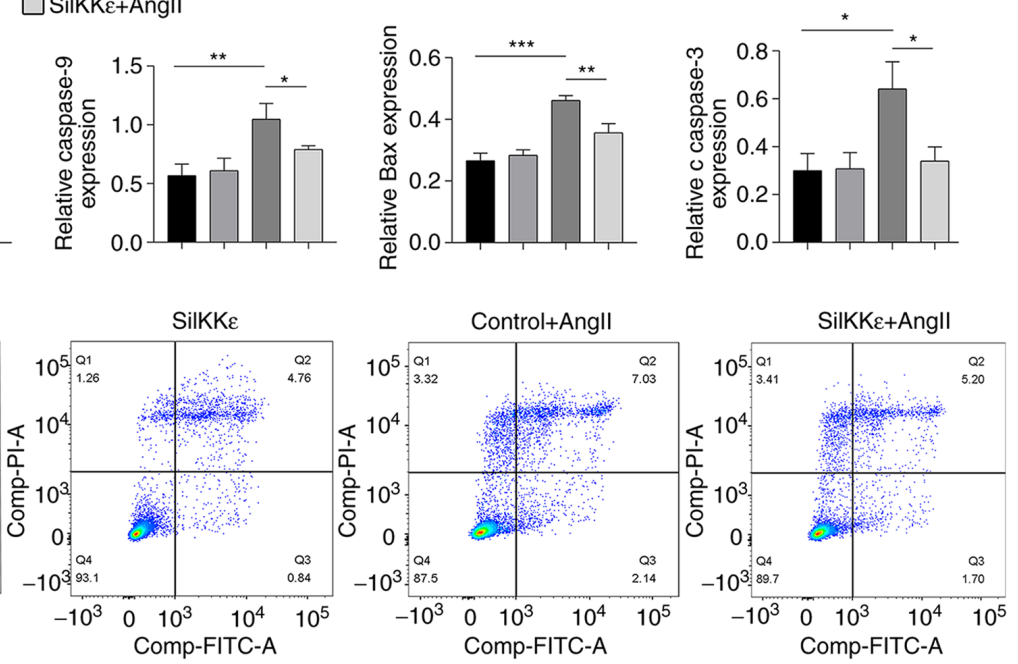

F $\square$ Control $\square$ Control+Angll

Control $\square$ Control+Angll $\square$ SilKKe $\square$ SilKKe+Angll
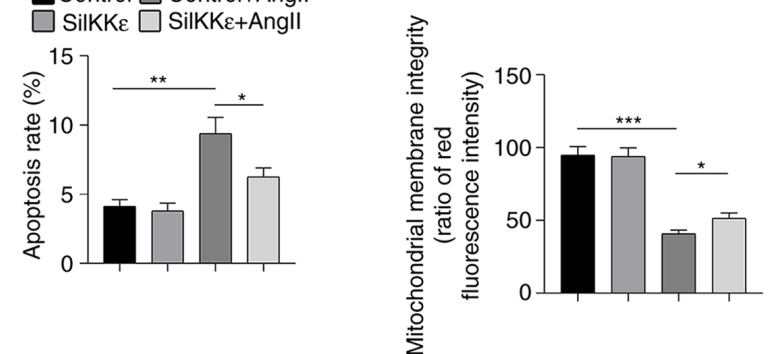

$\mathrm{E}$
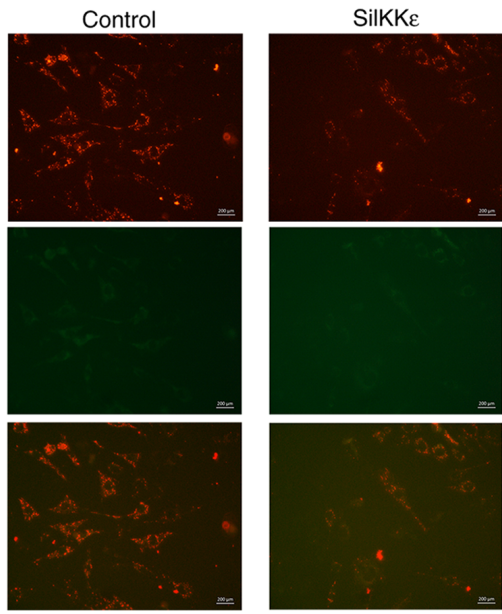

Control+Angll
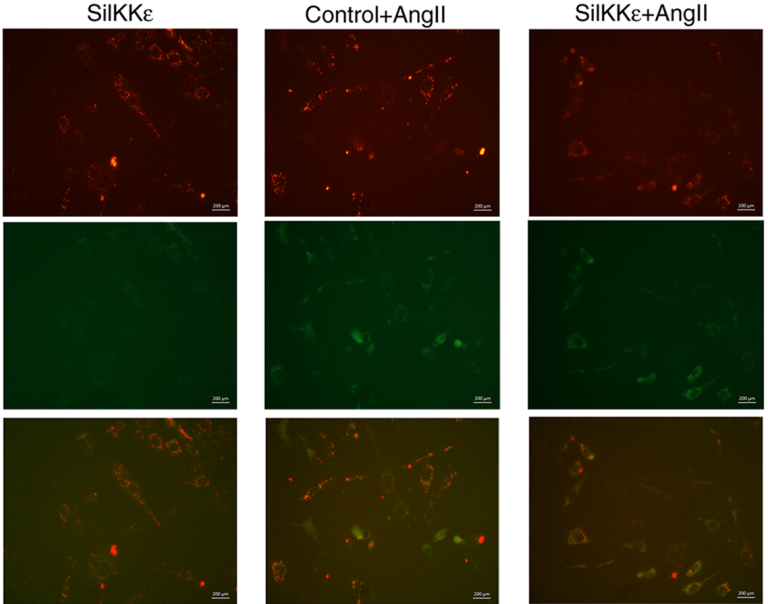

Figure 1. Knockdown of IKKe attenuates AngII-induced apoptosis in VSMCs. (A) Bax, caspase-9, c-caspase-3 and IKKe expression levels were detected via western blot analysis in AngII-induced VSMCs following siRNA transfection for $24 \mathrm{~h}$ ( $\mathrm{n}=4-5$ per experimental group). (B) Quantitative results of expression levels of Bax, caspase-9 and c-caspase-3 ( $\mathrm{n}=4-5$ per experimental group). (C and D) Quantitative flow cytometry results of the apoptosis rate of VCMCs exposed to AngII for $24 \mathrm{~h}$ ( $\mathrm{n}=4-5$ per experimental group). (E) Representative images and (F) quantitative results of JC-1 staining in VSMCs exposed to AngII for $24 \mathrm{~h}$ ( $\mathrm{n}=6$ per experimental group; magnification, $\mathrm{x} 400)$. Scale bar, $200 \mu \mathrm{m}$. Each experiment was repeated three times. ${ }^{*} \mathrm{P}<0.05 ;{ }^{* *} \mathrm{P}<0.01{ }^{*}{ }^{* * *} \mathrm{P}<0.001$. $\mathrm{IKK} \varepsilon$, inhibitor of nuclear factor- $\mathrm{kB}$ kinase subunit $\varepsilon$; AngII, angiotensin II; VSMCs, vascular smooth muscle cells; si, small interfering; c, cleaved. 
A
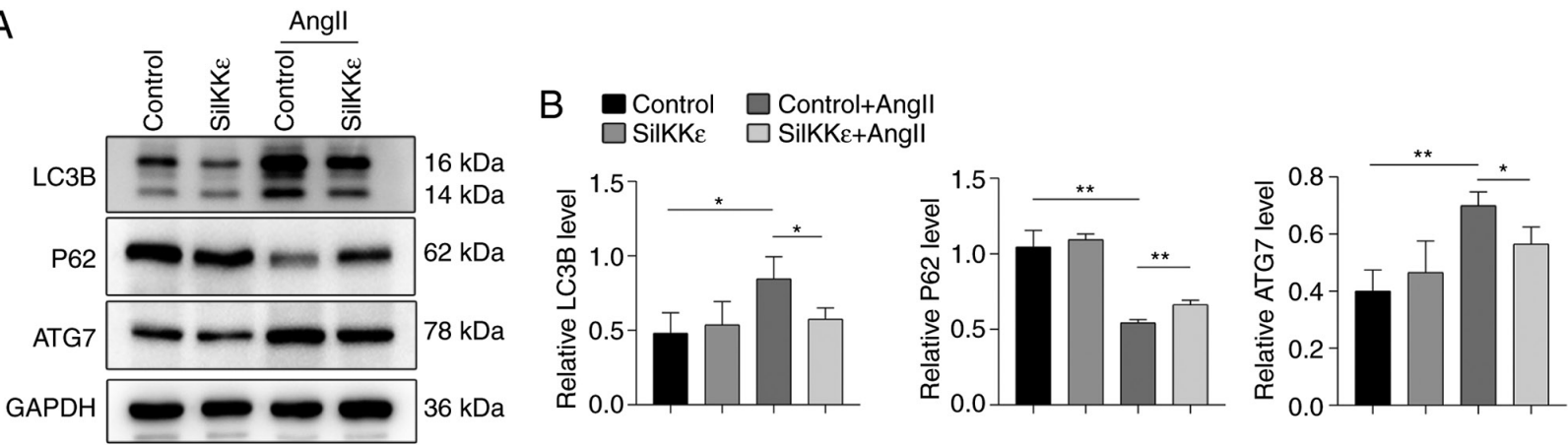

C

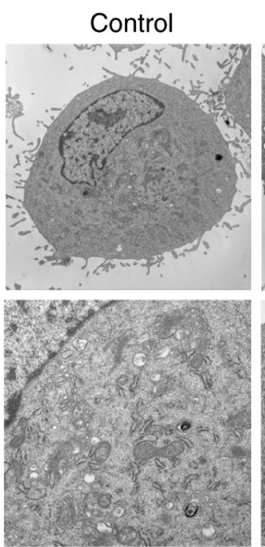

SilKKe

Control+Angll

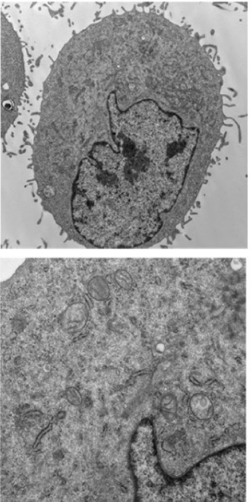

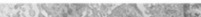
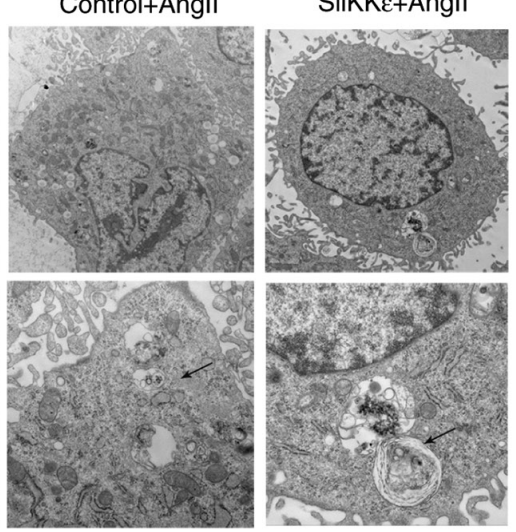

D $\square$ Control $\square$ Control+Angll
$\square$ SilKKe $\square$ SilKKe+Angll

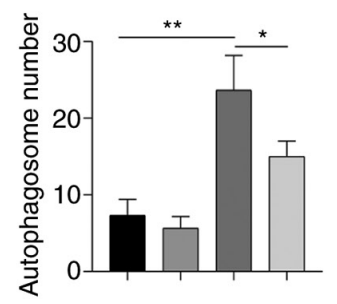

Figure 2. Knockdown of IKK $\varepsilon$ attenuates AngII-induced excessive autophagy in VSMCs. (A) LC3B, P62 and ATG7 expression levels detected via western blot analysis in AngII-induced VSMCs following siRNA transfection for $24 \mathrm{~h}$ ( $\mathrm{n}=4-5$ per experimental group). (B) Quantitative results of the expressions levels of LC3B, P62 and ATG7. (C) Representative transmission electron microscopy images and (D) autophagosome quantification in VSMCs exposed to AngII for $24 \mathrm{~h}(\mathrm{n}=4-5$ per experimental group; magnification, $\mathrm{x} 10,000)$. Each experiment was repeated three times. " $\mathrm{P}<0.05$; ${ }^{* *} \mathrm{P}<0.01$. IKKe, inhibitor of nuclear factor- $\mathrm{\kappa B}$ kinase subunit $\varepsilon$; AngII, angiotensin II; VSMCs, vascular smooth muscle cells; ATG7, autophagy-related protein 7; si, small interfering.

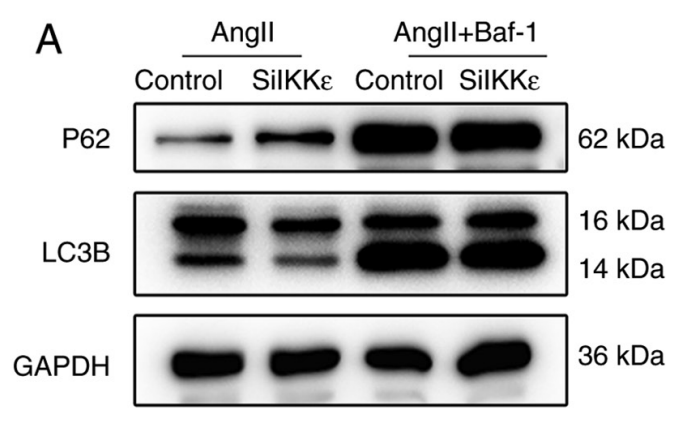

C

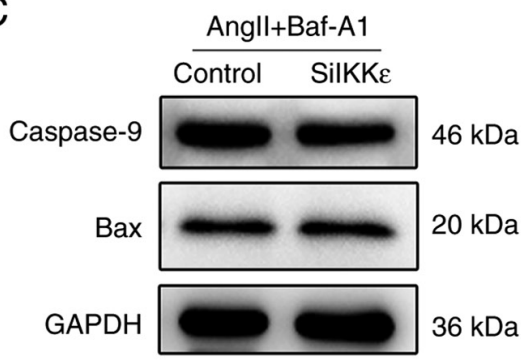

B
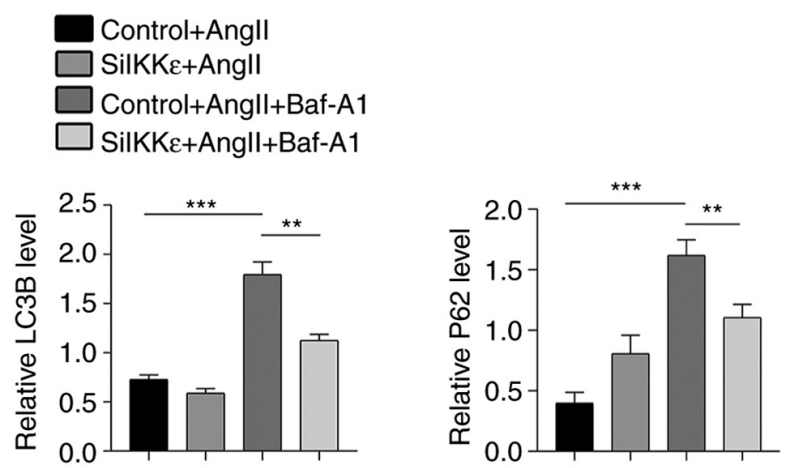

D Control+Angll+Baf-A1

SilKKe+Angll+Baf-A1

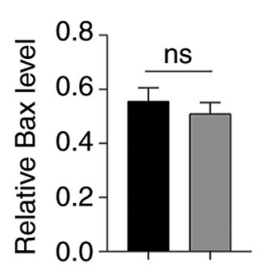

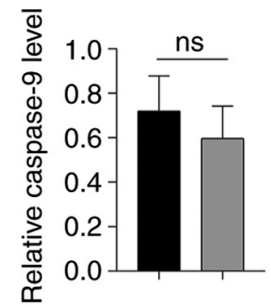

Figure 3. IKKe plays an important role in the association of autophagy and apoptosis. (A) Representative western blot images and (B) quantitative results of LC3B and P62 expression in VSMCs pretreated with Baf-A1 for $4 \mathrm{~h}$, followed by siRNA transfection and AngII-induction for $24 \mathrm{~h}$ ( $\mathrm{n}=4-5$ per experimental group). (C) Representative western blot images and (D) quantitative results of caspase-9 and Bax in VSMCs pretreated with Baf-A1 for $4 \mathrm{~h}$, followed by siRNA transfection and AngII-induction for $24 \mathrm{~h}\left(\mathrm{n}=4-5\right.$ per experimental group). Each experiment was repeated three times. ${ }^{* *} \mathrm{P}<0.01 ;{ }^{* * *} \mathrm{P}<0.001$. IKKe, inhibitor of nuclear factor- $\mathrm{\kappa B}$ kinase subunit $\varepsilon$; AngII, angiotensin II; VSMCs, vascular smooth muscle cells; si, small interfering; Baf-A1, bafilomycin A1; ns, not significant. 
A

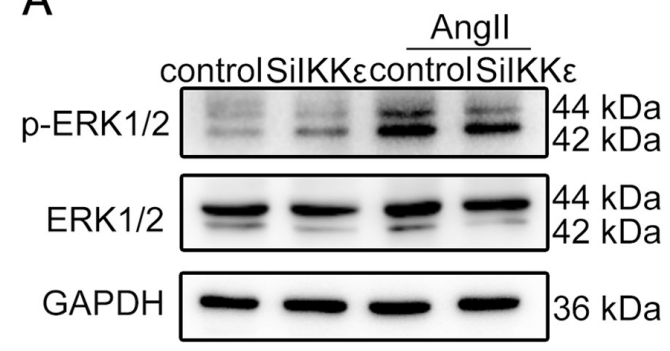

B control $\square$ control+Angll SilKKe $\square$ SilKKE+Angll

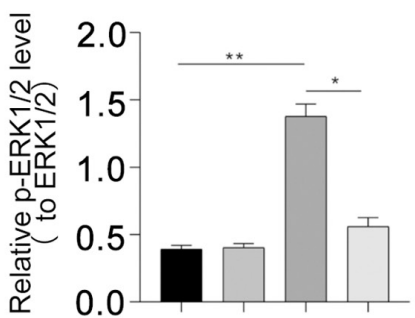

Figure 4. IKKe-regulated autophagy involves the ERK1/2 signaling pathway. (A) Representative western blot images and (B) quantitative results of phosphorylated and total protein levels of ERK1/2 following siRNA transfection in AngII-induced vascular smooth muscle cells for $24 \mathrm{~h}$ ( $\mathrm{n}=4-5 \mathrm{per}$ experimental group). Each experiment was repeated three times. ${ }^{*} \mathrm{P}<0.05 ;{ }^{* *} \mathrm{P}<0.01$. IKK $\varepsilon$, inhibitor of nuclear factor- $\kappa \mathrm{B}$ kinase subunit $\varepsilon$; si, small interfering; AngII, angiotensin II; p, phosphorylated.

Although previous findings have suggested that the IKK family plays an important role in autophagy (29), few studies have reported the role of IKKe in autophagy. A previous study demonstrated that IKKe played a protective role against cardiovascular diseases (30). Thus, inhibition of the autophagy of VSMCs may provide a novel treatment against AAA.

The autophagic flow plays an important role in autophagy (31). In the present study, autophagy was blocked with Baf-A1 to prevent the downstream events of autophagy by inhibiting lysosomal degradation (32). IKKع knockdown suppressed the synthesis of autophagy initiation-related autophagy vesicles and the accumulation of autophagosomes; therefore, it was hypothesized that IKKe played an important role in the early stages of autophagic flow in VSMCs.

Apoptosis and autophagy are important biological activities that contribute to the stability of the internal environment, thus promoting the organism's survival (33). Maintaining a balance between autophagy and apoptosis is critical for cell development (33). Abnormal induction of the autophagic flux has been indicated to promote apoptotic neuronal cell death (34). Autophagy has been demonstrated to play a protective role in attenuating AngII-induced oxidative stress and inflammation (35). However, autophagy has been indicated to contribute to apoptosis in cardiac microvascular endothelial cells (36). In the present study, treatment with Baf-A1 inhibited the expression levels of AngII-induced Bax and Caspase-9 in VSMCs. Therefore, it was hypothesized that IKKe deficiency reduced the apoptosis of VSMCs by decreasing autophagy to alleviate AAA formation.

The results of a previous study indicated that activation of ERK signaling is detected in a number of tumor cells, such as melanomas and gastric carcinoma (37). Furthermore, it has been reported that the phosphorylation of ERK induced during tumor development is dependent on IKKe (38). Continuous activation of the MEK/ERK signaling pathway directly induced autophagy (39) and induced apoptosis in tumor cells $(40,41)$. The findings of the present study revealed that the ERK1/2 pathway was activated in parallel with AngII-induced apoptosis in VSMCs, consistent with the findings generated from a previous study (11). The ERK1/2 pathway was possibly associated with AngII-induced autophagy. Furthermore, there was no significant difference in the phosphorylation of MEK1/2 and p38 in VSMCs after AngII treatment. Thus, the present study revealed that IKKe knockdown inhibited the phosphorylation of ERK1/2 in VSMCs.
In conclusion, the present study demonstrated that IKKe induced excessive autophagy in VSMCs, leading to increased apoptosis and potentially AAA formation. Therefore, inhibition of IKKe has the potential to act as a therapeutic target, to inhibit autophagy and apoptosis to reduce AAA occurrence.

Nonetheless, the present study was limited by several factors. The VSMC cell line used differs from primary cells, and the levels of IKKe were not increased by AngII treatment in the present study. The findings of the present study should be extended by analyzing both upstream and downstream mechanisms of IKKe and the ERK1/2 signaling pathway. Future studies should therefore focus on these shortcomings to provide more comprehensive results.

\section{Acknowledgements}

Not applicable.

\section{Funding}

The present study was supported by the National Natural Science Foundation of China (grant. No. 81870193), the Project of Invigorating Health Care through Science, Technology and Education, Jiangsu Provincial Key Medical Discipline (grant. no. ZDXKA2016021) and the Young Program of National Natural Science Foundation of China (grant. no. 81700415).

\section{Availability of data and materials}

The datasets used and/or analyzed during the current study are available from the corresponding author on reasonable request.

\section{Authors' contributions}

GC, YC, YX and YY performed the experiments. GC, YL and $\mathrm{HC}$ conducted data analysis. $\mathrm{YX}, \mathrm{WC}$ and $\mathrm{XC}$ designed the experiments and aided in data analyses. WC and XC confirm the authenticity of all the raw data. All authors have read and approved the final manuscript.

\section{Ethics approval and consent to participate}

Not applicable. 


\section{Patient consent for publication}

Not applicable.

\section{Competing interests}

The authors declare that they have no competing interests.

\section{References}

1. Brangsch J, Reimann C, Collettini F, Buchert R, Botnar RM and Makowski MR: Molecular imaging of abdominal aortic aneurysms. Trends Mol Med 23: 150-164, 2017.

2. Aggarwal S, Qamar A, Sharma V and Sharma A: Abdominal aortic aneurysm: A comprehensive review. Exp Clin Cardiol 16 $11-15,2011$

3. Laine MT, Laukontaus SJ, Sund R, Aho PS, Kantonen I, Albäck A and Venermo M: A population-based study of abdominal aortic aneurysm treatment in finland 2000 to 2014. Circulation 136 $1726-1734,2017$

4. Keisler B and Carter C: Abdominal aortic aneurysm. Am Fam Physician 91: 538-543, 2015.

5. Riches K, Angelini TG, Mudhar GS, Kaye J, Clark E, Bailey MA Sohrabi S, Korossis S, Walker PG, Scott DJ and Porter KE: Exploring smooth muscle phenotype and function in a bioreactor model of abdominal aortic aneurysm. J Transl Med 11: 208, 2013

6. Peshkova IO, Schaefer G and Koltsova EK: Atherosclerosis and aortic aneurysm-is inflammation a common denominator? FEBS J 283: 1636-1652, 2016

7. Stegbauer J, Thatcher SE, Yang G, Bottermann K, Rump LC, Daugherty A and Cassis LA: Mas receptor deficiency augments angiotensin II-induced atherosclerosis and aortic aneurysm ruptures in hypercholesterolemic male mice. J Vasc Surg 70: 1658-1668.e1, 2019.

8. Cao C, Zhu Y, Chen W, Li L, Qi Y, Wang X, Zhao Y, Wan X and Chen X: IIKKe knockout prevents high fat diet induced arterial atherosclerosis and NF- $\mathrm{NB}$ signaling in mice. PLoS One 8 : e64930, 2013.

9. Zhang J, Tian M, Xia Z and Feng P: Roles of IкB kinase $\varepsilon$ in the innate immune defense and beyond. Virol Sin 31: 457-465, 2016

10. Zhang L, Wang L, Chen W, Xu Y, Wang L, Iskandar R, Wang Y and Chen $\mathrm{X}$ : The expression of inhibitor of nuclear factor kappa-B kinase epsilon (IKKe) in human aortic aneurysm. Folia Morphol (Warsz) 76: 372-378, 2017.

11. Chai H, Tao Z, Qi Y, Qi H, Chen W, Xu Y, Zhang L, Chen H and Chen X: IKK epsilon deficiency attenuates angiotensin II-induced abdominal aortic aneurysm formation in mice by inhibiting inflammation, oxidative stress, and apoptosis. Oxid Med Cell Longev 2020: 3602824, 2020.

12. D'Arcy MS: Cell death: A review of the major forms of apoptosis, necrosis and autophagy. Cell Biol Int 43: 582-592, 2019.

13. Simon HU: Autophagy in myocardial differentiation and cardiac development. Circ Res 110: 524-525, 2012.

14. Song S, Tan J, Miao Y, Li M and Zhang Q: Crosstalk of autophagy and apoptosis: Involvement of the dual role of autophagy under ER stress. J Cell Physiol 232: 2977-2984, 2017.

15. Huang J, Wang T, Wright AC, Yang J, Zhou S, Li L, Yang J, Small A and Parmacek MS: Myocardin is required for maintenance of vascular and visceral smooth muscle homeostasis during postnatal development. Proc Natl Acad Sci USA 112: 4447-4452, 2015

16. Yu L, Alva A, Su H, Dutt P, Freundt E, Welsh S, Baehrecke EH and Lenardo MJ: Regulation of an ATG7-beclin 1 program of autophagic cell death by caspase-8. Science 304: 1500-1502, 2004

17. Leonardi M, Perna E, Tronnolone S, Colecchia D and Chiariello M: Activated kinase screening identifies the IKBKE oncogene as a positive regulator of autophagy. Autophagy 15: 312-326, 2019.

18. Ramadan A, Al-Omran M and Verma S: The putative role of autophagy in the pathogenesis of abdominal aortic aneurysms. Atherosclerosis 257: 288-296, 2017.

19. Petit PX, Lecoeur H, Zorn E, Dauguet C, Mignotte B and Gougeon ML: Alterations in mitochondrial structure and function are early events of dexamethasone-induced thymocyte apoptosis. J Cell Biol 130: 157-167, 1995.

20. Klionsky DJ, Abdelmohsen K, Abe A, Abedin MJ, Abeliovich H, Acevedo Arozena A, Adachi H, Adams CM, Adams PD, Adeli $\mathrm{K}$ et al: Guidelines for the use and interpretation of assays for monitoring autophagy (3rd edition). Autophagy 12: 1-222, 2016.
21. Daye D and Walker TG: Complications of endovascular aneurysm repair of the thoracic and abdominal aorta: Evaluation and management. Cardiovasc Diagn Ther 8 (Suppl 1): S138-S156, 2018

22. Gottlieb RA: Mitochondrial signaling in apoptosis: Mitochondrial daggers to the breaking heart. Basic Res Cardiol 98: 242-249, 2003

23. Lopez de Figueroa P, Lotz MK, Blanco FJ and Carames B Autophagy activation and protection from mitochondrial dysfunction in human chondrocytes. Arthritis Rheumatol 67: 966-976, 2015.

24. Kosacka J, Nowicki M, Paeschke S, Baum P, Bluher M and Kloting N: Up-regulated autophagy: As a protective factor in adipose tissue of WOKW rats with metabolic syndrome. Diabetol Metab Syndr 10: 13, 2018.

25. Xing H, Peng M, Li Z, Chen J, Zhang $\mathrm{H}$ and Teng X: Ammonia inhalation-mediated mir-202-5p leads to cardiac autophagy through PTEN/AKT/mTOR pathway. Chemosphere 235: 858-866, 2019.

26. Yang W, Duan Q, Zhu X, Tao K and Dong A: Follistatin-Like 1 attenuates ischemia/reperfusion injury in cardiomyocytes via regulation of autophagy. Biomed Res Int 2019: 9537382, 2019.

27. Zhang YY, Shi YN, Zhu N, Wang W, Deng CF, Xie XJ, Liao DF and Qin L: Autophagy: A killer or guardian of vascular smooth muscle cells. J Drug Target 28: 449-455, 2020.

28. Wang Z, Guo J, Han X, Xue M, Wang W, Mi L, Sheng Y, Ma C, Wu Jand Wu X:Metformin represses the pathophysiology of AAA by suppressing the activation of PI3K/AKT/mTOR/autophagy pathway in ApoE $\%$ mice. Cell Biosci 9: 68, 2019.

29. Criollo A, Senovilla L, Authier H, Maiuri MC, Morselli E, Vitale I, Kepp O, Tasdemir E, Galluzzi L, Shen S, et al: The IKK complex contributes to the induction of autophagy. EMBO J 29: 619-631, 2010.

30. Cao C, Zhu Y, Chen W, Li L, Qi Y, Wang X, Zhao Y, Wan X and Chen $\mathrm{X}$ : IKKe knockout prevents high fat diet induced arterial atherosclerosis and NF- $\mathrm{B}$ s signaling in Mice. PLoS One 8: e64930, 2013

31. Wu L, Duan Q, Gao D, Wang Y, Xue S, Li W and Lei M:Zearalenone blocks autophagy flow and induces cell apoptosis during embryo implantation in gilts. Toxicol Sci 175: 126-139, 2020.

32. Cheng Z, Zhang M, Hu J, Lin J, Feng X, Wang S, Wang T, Gao E, Wang $\mathrm{H}$ and Sun D: Mst1 knockout enhances cardiomyocyte autophagic flux to alleviate angiotensin II-induced cardiac injury independent of angiotensin II receptors. J Mol Cell Cardiol 125: 117-128, 2018.

33. Liu J, Liu W and Yang H: Balancing apoptosis and autophagy for Parkinson's disease therapy: Targeting BCL-2. ACS Chem Neurosci 10: 792-802, 2019.

34. Chung Y, Lee J, Jung S, Lee Y, Cho JW and Oh YJ: Dysregulated autophagy contributes to caspase-dependent neuronal apoptosis. Cell Death Dis 9: 1189, 2018.

35. Lu Y,LiS, Wu H,BianZ, Xu J,Gu C, Chen X and Yang D: Beneficial effects of astragaloside IV against angiotensin II-induced mitochondrial dysfunction in rat vascular smooth muscle cells. Int J Mol Med 36: 1223-1232, 2015.

36. Wang R, Yang Q, Wang X, Wang W, Li J, Zhu J, Liu X, Liu J and $\mathrm{Du}$ J: FoxO3 $\alpha$-mediated autophagy contributes to apoptosis in cardiac microvascular endothelial cells under hypoxia. Microvasc Res 104: 23-31, 2016.

37. Liu YL, Lai F, Wilmott JS, Yan XG, Liu XY, Luan Q, Guo ST, Jiang CC, Tseng HY, Scolyer RA, et al: Noxa upregulation by oncogenic activation of MEK/ERK through CREB promotes autophagy in human melanoma cells. Oncotarget 5: 11237-11251, 2014.

38. Goktuna SI, Shostak K, Chau TL, Heukamp LC, Hennuy B, Duong HQ, Ladang A, Close P, Klevernic I, Olivier F, et al: The prosurvival IKK-related kinase IKKe integrates LPS and IL17A signaling cascades to promote wnt-dependent tumor development in the intestine. Cancer Res 76: 2587-2599, 2016.

39. Zucchini-Pascal N, de Sousa G and Rahmani R: Lindane and cell death: At the crossroads between apoptosis, necrosis and autophagy. Toxicology 256: 32-41, 2009.

40. Wang X, Martindale JL and Holbrook NJ: Requirement for ERK activation in cisplatin-induced apoptosis. J Biol Chem 275: 39435-39443, 2000.

41. Cagnol S, Van Obberghen-Schilling EB and Chambard JC: Prolonged activation of ERK1,2 induces FADD-independent caspase 8 activation and cell death. Apoptosis 11: 337-346, 2006.

This work is licensed under a Creative Commons Attribution-NonCommercial-NoDerivatives 4.0 International (CC BY-NC-ND 4.0) License. 